\title{
Risk factors and molecular epidemiology of community-onset, multidrug resistance extend- ed-spectrum $\beta$-lactamase-producing Escherichia coli infections
}

\author{
So Yeon Park ${ }^{1,2}$, Cheol-In Kang ${ }^{2}$, Yu Mi Wi³, Doo Ryeon Chung ${ }^{2}$, Kyong Ran Peck ${ }^{2}$, Nam-Yong Lee ${ }^{4}$, and \\ Jae-Hoon Song ${ }^{2}$
}

${ }^{1}$ Division of Infectious Diseases, Hallym University Kangdong Sacred Heart Hospital, Seoul; ${ }^{2}$ Division of Infectious Diseases, Samsung Medical Center, Sungkyunkwan University School of Medicine, Seoul; ${ }^{3}$ Division of Infectious Diseases, Samsung Changwon Hospital, Sungkyunkwan University School of Medicine, Changwon; ${ }^{4}$ Department of Laboratory Medicine, Samsung Medical Center, Sungkyunkwan University School of Medicine, Seoul, Korea

Received: April 27, 2015 Revised : January 15, 2016 Accepted: January 22, 2016

\section{Correspondence to} Cheol-In Kang, M.D.

Division of Infectious Diseases, Samsung Medical Center, Sungkyunkwan University School of Medicine, 81 Irwon-ro, Gangnam$\mathrm{gu}$, Seoul 06351, Korea

Tel: +82-2-3410-0324

Fax: +82-2-3410-0064

E-mail: collacin@hotmail.com
Background/Aims: Although multidrug resistance (MDR) among extended-spectrum $\beta$-lactamase-producing Escherichia coli (ESBL-EC) poses significant therapeutic challenges, little is known regarding the risk factors and epidemiology of community-onset MDR-ESBL-EC infections. We performed this study to investigate risk factors and the molecular epidemiology of community-onset MDRESBL-EC infections.

Methods: We conducted a case-control-control study of community-onset infections. MDR-ESBL-EC was defined as ESBL-EC that demonstrated in vitro resistance to trimethoprim-sulfamethoxazole, fluoroquinolones (FQs), and gentamicin. Patients with MDR-ESBL-EC infections were designated as case patients. A control group I (CG I) patient was defined as a person whose clinical sample yielded ESBL-EC that did not meet the criteria for MDR. A control group II (CG II) patient was defined as a patient with a non-ESBL-EC infection.

Results: Of 108 patients with ESBL-EC infections, 30 cases (27.8\%) were due to MDR-ESBL-EC. Compared with CG I, prior use of FQs (odds ratio [OR], 3.16; 95\% confidence interval [CI], 1.11 to 8.98) and immunosuppressant use (OR, 10.47; 95\% CI, 1.07 to 102.57) were significantly associated with MDR-ESBL-EC. Compared with CG II, prior use of FQs (OR, 15.53; 95\% CI, 2.86 to 84.27) and healthcare-associated infection (OR, 5.98; 95\% CI, 2.26 to 15.86 ) were significantly associated with MDR-ESBL-EC. CTX-M-15 was the most common in MDR-ESBL-EC infections (59.1\% [13/22]), while CTX-M-14 was the most common in non-MDR-ESBL-EC infections (41.6\% [32/77]). CTX-M-15 was significantly associated with MDR-ESBLEC $(59.1 \%$ vs. $32.5 \%, p=0.028)$. Pulsed-field gel electrophoresis showed clonal diversity of MDR-ESBL-EC isolates.

Conclusions: The emergence of strains of MDR-ESBL-EC in the community poses an important new public health threat. More information on the emergence and transmission of these strains will be necessary in order to prevent their spread.

Keywords: Drug resistance, multiple; Extended-spectrum $\beta$-lactamase; Escherichia coli; Community-onset infection 


\section{INTRODUCTION}

Extended-spectrum $\beta$-lactamase-producing Escherichia coli (ESBL-EC) have become increasingly prevalent nosocomial pathogens [1-3]. During recent years, community-onset infections due to ESBL-EC have emerged worldwide $[4,5]$. ESBL-EC are also frequently resistant to non- $\beta$-lactam antibiotics, such as fluoroquinolones (FQs), trimethoprim-sulfamethoxazole (TMP-SMX), and aminoglycosides $[2,4]$. FQs, cephalosporins, and TMP-SMX are recommended as empirical therapies for community-onset infection due to E. coli [6-8]. Most patients with community-onset infections caused by these organisms have urinary tract infections (UTIs) or intra-abdominal infections $[5,9]$. FQs and TMP-SMX may be regarded as the treatment of choice or as alternative antimicrobial therapy for UTIs or intra-abdominal infections due to ESBL-EC, if the bacteria are susceptible in vitro to these agents [4].

The clinical relevance of multidrug resistance (MDR) among ESBL-EC (i.e., those resistant to multiple other antibiotics or antibiotic classes in addition to the oxymino $\beta$-lactams) is of great concern because therapeutic options are severely limited [10]. As the frequency of infection caused by MDR-ESBL-EC is increasing, so the use of carbapenems is rising, which may contribute to the spread of carbapenem resistance. Moreover, isolation of carbapenem resistant ESBL-producing Enterobacteriaceae has been increasingly reported [11]. In addition, empirical antibiotic treatment of community-onset infections may be inappropriate if MDR-ESBLEC are highly prevalent in the community.

Even though MDR-ESBL-EC poses significant therapeutic challenges, there is still little clinical information about community-onset MDR-ESBL-EC infections. Although several studies have reported on hospital-acquired MDR-ESBL-EC infections, data regarding community-onset MDR-ESBL-EC infections are limited $[10,12,13]$.

This study was conducted to determine risk factors and the molecular epidemiology of MDR among ESBL-EC causing community-onset infections. We wanted to identify risk factors associated with the acquisition of community-onset MDR-ESBL-EC infections. In addition, we wanted to identify risk factors associated with acquisition of a MDR-ESBL-EC among patients with community-onset ESBL-EC infections. For this purpose, we employed the case-control-control study design, which enables more accurate identification of risk factors for multidrug-resistant pathogens than the standard case-control study design [14]. Using data collected in a 9-month period at two university hospitals, we conducted parallel analyses in which patients who presented with an infection caused by MDR-ESBL-EC were compared to patients who presented with an infection caused by non-MDR-ESBL-EC and patients with non-ESBL-EC infections.

\section{METHODS}

\section{Study design and patients}

We performed a post hoc analysis of individual patients included in a previous study [15]. In the current study, a case-control-control design was used to evaluate risk factors associated with MDR-ESBL-EC in community-onset infections. Study subjects were prospectively identified as previously described [15] through a computerized database maintained by the clinical microbiology laboratory. The study was conducted between September 2010 and May 2011 at Samsung Medical Center in Seoul (a 1,950-bed tertiary care university hospital) and at Samsung Changwon Hospital (a 70o-bed community-based university-affiliated hospital in Changwon, Korea). Patients with community-onset infection due to E. coli were included in the study. The inclusion and exclusion criteria were the same as reported previously [15]. A case patient was defined as a person whose clinical sample yielded MDR-ESBL-EC. A control group I (CG I) patient was defined as a person whose clinical sample yielded ESBL-EC that did not meet the criteria for MDR. A control group II (CG II) patient was defined as a patient with a non-ESBL-EC infection. For each patient who presented with an infection caused by ESBL-EC, one CG II was randomly chosen from a group of outpatients from whom non-ESBL-EC had been isolated from a clinical sample that had been sent to the same laboratory for culturing during the week following presentation of the case patient. The CG II patients were matched according to the culture specimen and acquisition unit. The patient data collected included age, sex, underlying disease, severity of underlying diseases as 
classified by McCabe and Jackson criteria [16], presence of severe sepsis or septic shock at presentation, severity of illness at presentation according to the Pitt bacteremia score [2], and receipt of any antimicrobial therapy prior to onset of the infection. The presence of any of the following comorbid conditions was also documented: neutropenia, recent surgical procedure within the prior 3 months, corticosteroid use within the prior 1 month, immunosuppressive therapy within the prior 1 month, or the presence of an indwelling urinary catheter or percutaneous tube. Because the study was observational, the attending physician decided on indications for cultures, other tests, and treatments based on each patient's individual clinical situation. The study was approved by the Institutional Review Board of Samsung Medical Center, Seoul, Korea. Written informed consent was not required because of the observational nature of the study.

\section{Definitions}

The community-onset infections were further classified as healthcare-associated (HCA) or community-associated(CA). Episodes were considered to be HCA if any of the following criteria were present: a history of a 48 hours or longer hospital admission in the previous 90 days, hemodialysis, intravenous medication, home wound care in the previous 30 days, or residence in a nursing home or long-term care facility $[9,17]$. Community-onset infections that did not meet these criteria were considered to be CA. MDR-ESBL-EC was defined as an ESBL-EC that demonstrated in vitro resistance to all of the following three antibiotics or antibiotic classes: TMP-SMX, FQs (i.e., ciprofloxacin and levofloxacin), and gentamicin. Non-MDR-ESBL-EC was defined as ESBL-EC that did not meet the criteria for MDR. Community-onset infection was defined as an infection diagnosed within the first 48 hours of hospitalization. The sites of infection were determined by the physicians on the basis of clinical evaluation and the isolation of EC from the presumed portal of entry [9,18]. Primary bacteremia was defined according to Centers for Disease Control and Prevention definitions [18]. Patients with immunosuppression included those who were on immunosuppressive therapy (chemotherapeutic agents, immunosuppressive agents, or radiation therapy). Neutropenia was defined as an absolute neutrophil count $<500$ neutrophils $/ \mathrm{mm}^{3}$. Sepsis was defined as the presence of a systemic inflammatory response syndrome (any two of the following: tachypnea $>20$ breaths per minute, white blood cell count $<4,000$ or $>12,000$ cells $/ \mu \mathrm{L}$, heart rate $>90$ beats per minute, and fever $>38.0^{\circ} \mathrm{C}$ or hypothermia $<36.0^{\circ} \mathrm{C}$ ) in addition to a documented or presumed infection [19]. Severe sepsis was defined as sepsis with one or more clinical signs of organ dysfunction and septic shock defined as sepsis with hypotension despite adequate fluid resuscitation along with the presence of perfusion abnormalities [19]. Prior antibiotic therapy was defined as the receipt of any systemic antibiotics for more than 48 hours in the preceding 30 days.

\section{Microbiologic analysis}

Identification of microorganisms, antimicrobial susceptibility testing, and ESBL confirmatory testing were performed as previously described [15]. Quality control was performed using the strains E. coli 25922 and Pseudomonas aeruginosa 27853 .

\section{Detection of ESBL genes and sequencing of the polymerase chain reaction product}

For the characterization of ESBL types, polymerase chain reactions (PCRs) and sequencing of PCR products were performed using available stored isolates, as described previously [15]. ESBL-related genes, such as TEM, SHV, CTX-M, and OXA, were amplified by PCR from clinical isolates as described in previous studies $[20,21]$. The types of ESBL genes were identified by comparing the sequences to those in the database of G. Jacoby and K. Bush (http//lahey.org/Studies/).

\section{Genetic typing by pulsed-field gel electrophoresis} Available MDR-ESBL-EC isolates were evaluated for genetic relatedness by pulsed-field gel electrophoresis (PFGE). For PFGE, agarose-embedded bacterial genomic DNA was digested with $20 \mathrm{U}$ Xba 1 . The restriction fragments were separated by electrophoresis in $0.5 \times$ Tris/borate/ethylenediaminetetraacetic acid (TBE) buffer. The subsequent PFGE analyses were performed on a CHEFMAPPER XA apparatus (Bio-Rad Laboratories, Hercules, CA, USA) as described previously [22]. The isolates were considered genetically related if the Dice coefficient correlation was $80 \%$ or greater, which corresponds to the possibly related criterion of Tenover et al. [23]. 


\section{Statistical analysis}

The Student $t$ test or the Mann-Whitney test was used to compare continuous variables, and the chi-square or Fisher exact test was used to compare categorical variables. We used a backward stepwise logistic regression analysis to control for the effects of confounding variables. All risk factors with a $p<0.1$ at the bivariate level were included in the multivariate logistic model predicting MDR-ESBL-EC infection. All variables for which the $p$ value was less than 0.05 in the multivariate analysis were retained in the final model. Interactions between variables were not introduced into the models. Odds ratios (ORs) and their 95\% confidence intervals (CIs) were calculated. All $p$ values were two-tailed, and $p<0.05$ was considered statistically significant. IBM SPSS version 19 (IBM Co., Armonk, NY, USA) was used for these analyses.

\section{RESULTS}

\section{Clinical characteristics and risk factors for commu- nity-onset infections caused by MDR-ESBL-EC}

During the study period, a total of 108 patients with community-onset ESBL-EC infections were identified and all of them were included in this study. Of the 108 isolates, $30(27.8 \%)$ were due to MDR-ESBL-EC. The mean age ( \pm standard deviation) of the study population was $62.4 \pm 16.0$ years, and $70(64.8 \%)$ were women. The most common underlying disease was diabetes mellitus (26.9\% [28/108]) and the most common site of infection was the urinary tract $(67.6 \%$ [73/108]). To analyze risk factors associated with community-onset MDR-ESBL-EC, data from the 30 patients with MDR-ESBL-EC were compared to those of the 78 patients with non-MDRESBL-EC (CG I) and 100 patients with non-ESBL-EC infections (CG II). The clinical characteristics of the case and control groups and risk factors for community-onset MDR-ESBL-EC infections are summarized in Table 1. Variables such as age, sex, underlying disease, and severity of illness at presentation were similar between the case and control groups. The source of isolates did not differ significantly between case and control groups. Compared with CG I, significant factors associated with community-onset MDR-ESBL-EC infections were immunosuppressive therapy and prior receipt of FQs (all $p<0.05)$. Compared with CG II, HCA infection, severity of illness at presentation, immunosuppressive therapy, and prior receipt of antibiotics, especially FQs, were significantly associated with MDR-ESBL-EC infections.

To identify independent risk factors associated with community-onset MDR-ESBL-EC infections, a logistic regression analysis was performed including variables with an association of $p<0.1$ at the univariate level. The independent risk factors associated with communityonset MDR-ESBL-EC infections are shown in Table 2. Immunosuppressive therapy (OR, 10.47; 95\% CI, 1.07 to 12.05; $p=0.044$ in model 1 ), prior receipt of FQs (OR, 3.16; 95\% CI, 1.11 to 8.98; $p=0.31$ in model 1; OR, 15.53; 95\% CI, 2.86 to $84.27 ; p=0.01$ in model 2 ), and HCA infection (OR, 5.98; 95\% CI, 2.26 to 15.86; $p<0.01$ in model 2) were found to be independent factors associated with community-onset MDR-ESBL-EC infections.

\section{Microbiological characteristics of MDR-ESBL-EC causing community-onset infections}

The 108 community-onset ESBL-EC isolates demonstrated variable resistance to other antibiotics. The prevalence of resistance to piperacillin/tazobactam (36.7\% vs. $29.5 \%, p=0.472)$ and amoxicillin/clavulanic acid (73.3\% vs. $59.0 \%, p=0.189$ ) were similar for the MDR-ESBL-EC group and the non-MDR-ESBL-EC group. However, strains resistant to tobramycin were more frequent in the MDR-ESBL-EC group than the non-MDR-ESBL-EC group (93.3\% vs. $44.9 \%, p<0.001$ ) (Table 3). None of the strains were resistant to imipenem or meropenem. Both MDR- and non-MDR-ESBL-EC isolates were highly susceptible to amikacin ( $100 \%$ vs. $98.7 \%, p=1.000$ ). Ninetynine isolates of ESBL-EC were available for further microbiological study. All of these isolates produced ESBLs from the CTX-M family. We compared MDR-ESBL-EC with non-MDR-ESBL-EC and the distribution of ESBL genes is summarized in Table 4. CTX-M-15 was the most common type of MDR-ESBL-EC (59.1\% [13/22]), while CTX-M-14 was the most common non-MDR-ESBL-EC (41.6\% [32/77]). CTX-M-15 was significantly associated with MDR-ESBL-EC (59.1\% vs. 32.5\%, $p=0.028$ ).

PFGE of 22 MDR-ESBL-EC strains was performed to characterize the clonality of these isolates. Because two isolates (test number 1 and 19) among the 22 MDR-ESBL-EC did not provide DNA bands by the PFGE method used in this study, we analyzed only 20 isolates. Applying a similarity index of $80 \%$ to the PFGE results, four 
Table 1. Univariate analysis of risk factors for community-onset multidrug-resistant extended-spectrum $\beta$-lactamase-producing Escherichia coli infections

\begin{tabular}{|c|c|c|c|c|c|}
\hline Risk factor & $\begin{array}{l}\text { Case group } \\
\qquad(\mathrm{n}=30)\end{array}$ & $\begin{array}{l}\text { Control group } \mathrm{I}^{\mathrm{a}} \\
(\mathrm{n}=78)\end{array}$ & $p$ value & $\begin{array}{l}\text { Control group } \mathrm{II}^{\mathrm{b}} \\
(\mathrm{n}=100)\end{array}$ & $p$ value \\
\hline Female sex & $22(73 \cdot 3)$ & $48(61.5)$ & 0.250 & $79(79.0)$ & 0.513 \\
\hline Older age ( $\geq 65 \mathrm{yr}$ ) & $17(56.7)$ & $39(50.0)$ & 0.535 & $49(49.0)$ & 0.461 \\
\hline Healthcare-associated infection & $20(66.7)$ & $36(46.2)$ & 0.056 & $20(20.0)$ & $<0.001$ \\
\hline \multicolumn{6}{|l|}{ Underlying disease } \\
\hline Diabetes mellitus & $8(26.7)$ & $20(25 \cdot 6)$ & 0.913 & $24(24 \cdot 0)$ & 0.766 \\
\hline Liver disease & $4(13 \cdot 3)$ & $9(11.5)$ & 0.752 & $10(10.0)$ & 0.737 \\
\hline Renal disease & $6(20.0)$ & $8(10.3)$ & 0.206 & $15(15 \cdot 0)$ & 0.573 \\
\hline Solid tumor & $6(20.0)$ & $18(23.1)$ & 0.730 & $20(20.0)$ & 1.000 \\
\hline Neurologic disease & $6(20.0)$ & $15(19.2)$ & 0.928 & $10(10.0)$ & 0.201 \\
\hline \multicolumn{6}{|c|}{ Severity of underlying illness by McCabe and Jackson criteria } \\
\hline Non-fatal & $18(60.0)$ & $58(74 \cdot 4)$ & 0.218 & $85(85.0)$ & \\
\hline Ultimately fatal & $11(36.7)$ & $16(20.5)$ & & $14(14 \cdot 0)$ & 0.012 \\
\hline Rapidly fatal & $1(3 \cdot 3)$ & $4(5.1)$ & & $1(1.0)$ & \\
\hline \multicolumn{6}{|l|}{ Comorbid condition } \\
\hline Recent operation & $1(3 \cdot 3)$ & $5(6.4)$ & 1.000 & $3(3.0)$ & 1.000 \\
\hline Neutropenia & $3(10.0)$ & $2(2.6)$ & 0.130 & $2(2.0)$ & 0.080 \\
\hline Immunosuppressive therapy & $4(13 \cdot 3)$ & $1(1.3)$ & 0.020 & $3(3.0)$ & 0.049 \\
\hline Central venous catheterization & $2(6.7)$ & $4(5 \cdot 1)$ & 0.669 & $3(3.0)$ & 0.326 \\
\hline Indwelling urinary catheter & $1(3 \cdot 3)$ & $7(9.0)$ & 0.439 & $5(5 \cdot 0)$ & 1.000 \\
\hline Percutaneous tube & $5(16.7)$ & $6(7 \cdot 7)$ & 0.175 & $5(5.0)$ & 0.050 \\
\hline Prior receipt of antibiotics & $13(43 \cdot 3)$ & $26(33 \cdot 3)$ & 0.333 & $8(8.0)$ & $<0.001$ \\
\hline Cephalosporin & $4(13 \cdot 3)$ & $14(17 \cdot 9)$ & 0.564 & $4(4 \cdot 0)$ & 0.082 \\
\hline$\beta$-Lactam or $\beta$-lactam $/ \beta$-lactamase inhibitor & $1(3 \cdot 3)$ & $7(9.0)$ & 0.439 & $1(1.0)$ & 0.410 \\
\hline Fluoroquinolone & $10(33 \cdot 3)$ & $10(12.8)$ & 0.014 & $2(2.0)$ & $<0.001$ \\
\hline \multicolumn{6}{|l|}{ Severity of illness } \\
\hline No sepsis & $20(66.7)$ & $51(65.4)$ & 0.611 & $66(66.0)$ & 0.671 \\
\hline Sepsis & $5(16.7)$ & $18(23.1)$ & & $22(22.0)$ & \\
\hline Severe sepsis & $1(3 \cdot 3)$ & $4(5 \cdot 1)$ & & $5(5.0)$ & \\
\hline Septic shock & $4(13 \cdot 3)$ & $5(6.4)$ & & $7(7.0)$ & \\
\hline \multicolumn{6}{|l|}{ Site of infection } \\
\hline Urinary tract & $20(66.7)$ & $53(67.9)$ & 1.000 & $67(67.0)$ & 1.000 \\
\hline Pancreaticobiliary tract & $2(6.7)$ & $10(12.8)$ & 0.504 & $11(11.0)$ & 0.731 \\
\hline Intra-abdomen & $5(16.7)$ & $5(6.4)$ & 0.137 & $7(7 \cdot 0)$ & 0.146 \\
\hline Unknown & $2(6.7)$ & $3(3.8)$ & 0.616 & $3(3.0)$ & 0.326 \\
\hline
\end{tabular}

Values are presented as number (\%).

${ }^{\text {a } C o n t r o l ~ g r o u p ~ I: ~ t h o s e ~ w h o s e ~ c l i n i c a l ~ s a m p l e ~ y i e l d e d ~ e x t e n d e d-s p e c t r u m ~} \beta$-lactamase-producing Escherichia coli (ESBL-EC) that did not meet the criteria for multidrug resistance.

${ }^{\mathrm{b}}$ Control group II: those with non-ESBL-EC infections. 
Table 2. Independent risk factors for multidrug-resistance in community-onset infections caused by extended-spectrum $\beta$-lactamase-producing Escherichia coli

\begin{tabular}{lcr}
\hline Risk factor & Adjusted OR (95\% CI) & $p$ value \\
\hline Model 1 (with control group I) & & 0.044 \\
Immunosuppressive therapy & $10.47(1.07-102.57)$ & 0.031 \\
Prior receipt of fluoroquinolone & $3.16(1.11-8.98)$ & 0.001 \\
Model 2 (with control group II) & & $<0.001$ \\
\hline Prior receipt of fluoroquinolone & $15.53(2.86-84.27)$ & $5.98(2.26-15.86)$ \\
Healthcare-associated infection & & \\
\hline
\end{tabular}

OR, odds ratio; CI, confidence interval.

Table 3. Comparison of rates of resistance to antimicrobials of Escherichia coli isolates causing community-onset infections ${ }^{\mathrm{a}}$

\begin{tabular}{|c|c|c|c|c|c|}
\hline \multirow[b]{2}{*}{ Antibiotic } & \multicolumn{5}{|c|}{$\%$ of patients (no. taking drug/total) } \\
\hline & $\begin{array}{l}\text { MDR-ESBL-EC } \\
\text { group }\end{array}$ & $\begin{array}{c}\text { Non-MDR- } \\
\text { ESBL-EC group }\end{array}$ & $p$ value & $\begin{array}{l}\text { Non-ESBL-EC } \\
\text { group }\end{array}$ & $p$ value \\
\hline Amoxicillin-clavulanate & $73.3(22 / 30)$ & $59.0(46 / 78)$ & 0.189 & $11.0(11 / 100)$ & $<0.001$ \\
\hline Piperacillin-tazobactam & $36.7(11 / 30)$ & $29 \cdot 5(23 / 78)$ & 0.472 & $11.0(11 / 100)$ & 0.001 \\
\hline Amikacin & $0.0(0 / 30)$ & $1.3(1 / 78)$ & 1.000 & $0.0(0 / 30)$ & \\
\hline Gentamicin & $100.0(30 / 30)$ & $38.5(30 / 78)$ & $<0.001$ & $20.0(20 / 100)$ & $<0.001$ \\
\hline Tobramycin & $93.3(28 / 30)$ & $44 \cdot 9(33 / 78)$ & $<0.001$ & $16.0(16 / 100)$ & $<0.001$ \\
\hline $\begin{array}{l}\text { Trimethoprim- } \\
\text { sulfamethoxazole }\end{array}$ & $100.0(30 / 30)$ & $34.6(27 / 78)$ & $<0.001$ & $22.0(22 / 100)$ & $<0.001$ \\
\hline Levofloxacin & $100.0(30 / 30)$ & $71.8(56 / 78)$ & $<0.001$ & $26.0(26 / 100)$ & $<0.001$ \\
\hline
\end{tabular}

MDR, multidrug resistance; ESBL-EC, extended-spectrum $\beta$-lactamase-producing Escherichia coli.

${ }^{a}$ According to automatic susceptibility testing.

Table 4. Comparison of ESBL types between MDR-ESBL-EC and non-MDR-ESBL-EC isolates

\begin{tabular}{lccc}
\hline Variable & MDR-ESBL-EC $(\mathrm{n}=22)$ & Non-MDR-ESBL-EC $(\mathrm{n}=77)$ & p value \\
\hline CTX-M-14 & $7(31.8)$ & $32(41.6)$ & 0.466 \\
CTX-M-15 & $13(59.1)$ & $25(32.5)$ & 0.028 \\
CTX-M-14 and CTX-M-15 & $2(9.1)$ & $1(1.3)$ & 0.123 \\
Other CTX-M type $^{\mathrm{a}}$ & 0 & $19(24.7)$ & 0.006 \\
\hline
\end{tabular}

Values are presented as number (\%).

ESBL-EC, extended-spectrum $\beta$-lactamase-producing Escherichia coli; MDR, multidrug resistance.

aOther CTX-M: CTX-M-24, 2 isolates; CTX-M-27, 10 isolates; CTX-M-3, 1 isolate; CTX-M-57, 6 isolates.

clonal clusters were identified, consisting of two or three isolates for each cluster. The 10 remaining isolates showed greater clonal diversity (Fig. 1). The microbiological characteristics of MDR-ESBL-EC that were available for further microbiological evaluation are shown in Table 5.

\section{DISCUSSION}

Our data showed that $27.8 \%$ of community-onset ESBL-EC isolates were MDR and significant risk factors for MDR among ESBL-EC isolates causing community-onset infections were prior exposure to FQs, receipt of immunosuppressive therapy, and HCA infection. Previous 


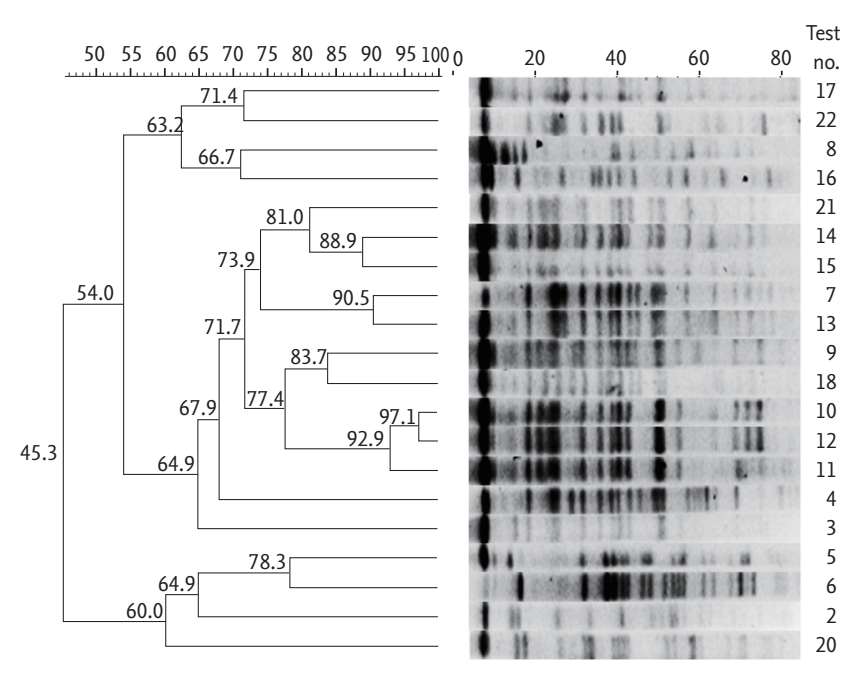

Figure 1. Dendrogram showing the relatedness of pulsedfield gel electrophoresis banding patterns for 20 multidrug resistance extended-spectrum $\beta$-lactamase-producing Escherichia coli isolates. Similarity among profiles was determined by cluster analysis in the dendrogram using a cutoff of at least $80 \%$.

studies have suggested that the prevalence of community-onset ESBL-EC infections varies widely by geographic region, and most ESBL-producing isolates show co-resistance to multiple antibiotics [5,9,24,25]. However, data on risk factors for community-onset MDR-ESBL-EC infections are limited. Although two previous studies evaluated risk factors for infections by MDR-ESBL-EC, these studies included mainly nosocomial infections and the proportion of community-onset infections was less than $10 \%$ of the study population $[10,13]$. To the best of our knowledge, this is the first study to investigate risk factors and molecular epidemiology of MDR-ESBLEC isolates among community-onset infections.

In the current study, we sought to identify risk factors for MDR-ESBL-EC among community-onset infections. The rate of MDR among ESBL-EC isolates was similar to rates found in previous studies $[10,12]$. We found that the receipt of immunosuppressive therapy, prior exposure to FQs, and HCA infection were associated with community-onset MDR-ESBL-EC infections. Patients with community-onset infection due to an MDR organism were more likely to be prescribed inactive empirical therapy. The MDR-ESBL-EC group received more inappropriate empirical therapy than the non-MDR-ESBLEC group (90.0\% vs. 66.7\%, $p=0.015$ ). Patients on immunosuppressive therapy, those with prior exposure to
FQs, and those with HCA infections should be offered carbapenems as empirical therapy even for communityonset infections.

In our previous study, prior receipt of FQs was an independent risk factor for nosocomial MDR-ESBL-EC and Klebsiella pneumoniae infections [12]. In our previous studies, HCA infection and prior receipt of FQs were associated with community-onset ESBL-EC infections [15]. In other previous studies, FQ use has been associated with the acquisition of other MDR gram-negative infections, such as P. aeruginosa and Acinetobacter baumannii $[26,27]$. In addition, Villers et al. [27] showed that the institution of a policy restricting the use of intravenous FQs could decrease the rate of infection with A. baumannii. Interestingly, a recent study showed a correlation between FQ resistance and MDR in ESBL-EC infections [28]. Because this has been previously reported for MDR gram-negative infections $[26,29,30]$, we can speculate that prior receipt of a FQ either results in selective pressure or facilitates the activation of intrinsic mechanisms that confer resistance to multiple antibiotic drug classes, such as the drug efflux mechanism.

In our study, immunosuppressive therapy was also associated with community-onset MDR-ESBL-EC infections. Immunosuppression presents an especially troublesome situation for critically ill patients as it has been identified as an independent risk factor for infectious morbidity and mortality [31]. Not surprisingly, a significantly greater proportion of immunosuppressed patients received antibiotic therapy during their treatment. Patients treated with immunosuppressive agents had more exposure to antibiotics than immunocompetent patients, which might be related to their acquiring MDR-ESBL-EC infections.

We observed CTX-M-15 was significantly associated with MDR-ESBL-EC (59.1\% vs. $32.5 \%, p=0.028)$. In recent studies, CTX-M-14 and CTX-M-15 were the predominant types of ESBLs, and CTX-M-15 was significantly associated with ST131 [32,33]. ST131 E. coli isolates had a trend toward greater nonsusceptibility to ciprofloxacin and cefepime [32,33]. Therefore, our data suggests that CTX-M-15 isolates show more MDR patterns than CTX-M-14.

We performed PFGE to examine the molecular epidemiology of community-onset MDR-ESBL-EC. Ten isolates among 20 MDR-ESBL-EC were not clonally 
Table 5. Microbiological characteristics of community-onset multidrug-resistant extended-spectrum $\beta$-lactamase-producing Escherichia coli available for further microbiological studies

\begin{tabular}{|c|c|c|c|c|c|c|c|c|c|}
\hline $\begin{array}{l}\text { Test } \\
\text { no. }\end{array}$ & Center & Department & $\begin{array}{l}\text { Speci- } \\
\text { men }\end{array}$ & $\begin{array}{l}\text { Date of } \\
\text { culture }\end{array}$ & $\begin{array}{l}\text { Cefepime } \\
\text { MIC }\end{array}$ & $\begin{array}{c}\text { Piperacillin/ } \\
\text { tazobactam } \\
\text { MIC }\end{array}$ & $\begin{array}{c}\text { Ciprofloxacin } \\
\text { MIC }\end{array}$ & $\begin{array}{c}\text { Ertapenem } \\
\text { MIC }\end{array}$ & ESBL types \\
\hline $1^{a}$ & SMC & ED & Urine & $2010-10-04$ & 8 & $8 / 4$ & $>128$ & $\leq 0.03$ & CTX-M-14 \\
\hline 2 & SMC & ED & Blood & $2010-10-20$ & 64 & $2 / 4$ & 8 & $\leq 0.03$ & CTX-M-14 \\
\hline 3 & SMC & ED & Blood & $2011-02-10$ & 128 & $8 / 4$ & $>128$ & 0.06 & CTX-M-15 \\
\hline 4 & SMC & ED & Blood & $2011-02-15$ & $>128$ & $16 / 4$ & 128 & 0.25 & CTX-M-15 \\
\hline 5 & SMC & ED & Urine & $2011-02-28$ & 16 & $2 / 4$ & 4 & $\leq 0.03$ & CTX-M-14 \\
\hline 6 & SMC & ED & Urine & 2011-03-15 & 32 & $8 / 4$ & $>128$ & 0.06 & CTX-M-15 \\
\hline 7 & SMC & ED & Blood & 2011-04-16 & 4 & $4 / 4$ & 32 & $\leq 0.03$ & CTX-M-14 \\
\hline 8 & SMC & OPD & Urine & $2011-05-18$ & $>128$ & $8 / 4$ & 64 & 0.12 & CTX-M-15 \\
\hline 9 & SMC & OPD & Urine & $2011-05-31$ & $>128$ & $4 / 4$ & 64 & 0.12 & CTX-M-15 \\
\hline 10 & $\mathrm{SCH}$ & ED & Blood & 2010-10-09 & $>128$ & $8 / 4$ & 64 & 0.06 & $\begin{array}{l}\text { CTX-M-14, } \\
\text { CTX-M-15 }\end{array}$ \\
\hline 11 & $\mathrm{SCH}$ & ED & Blood & $2010-10-10$ & $>128$ & $8 / 4$ & $>128$ & $\leq 0.03$ & CTX-M-15 \\
\hline 12 & $\mathrm{SCH}$ & OPD & Blood & $2010-10-15$ & $>128$ & $16 / 4$ & 64 & $\leq 0.03$ & $\begin{array}{l}\text { CTX-M-14, } \\
\text { CTX-M-15 }\end{array}$ \\
\hline 13 & $\mathrm{SCH}$ & OPD & Urine & $2010-10-18$ & 4 & $2 / 4$ & 32 & $\leq 0.03$ & CTX-M-15 \\
\hline 14 & $\mathrm{SCH}$ & ED & Blood & $2010-10-18$ & 64 & $4 / 4$ & $>128$ & $\leq 0.03$ & CTX-M-15 \\
\hline 15 & $\mathrm{SCH}$ & ED & Blood & $2010-12-11$ & 128 & $2 / 42 / 4$ & 64 & 0.06 & CTX-M-15 \\
\hline 16 & $\mathrm{SCH}$ & OPD & Urine & $2010-12-27$ & $>128$ & $32 / 4$ & $>128$ & 0.25 & CTX-M-15 \\
\hline 17 & $\mathrm{SCH}$ & ED & Urine & 2011-01-27 & 128 & $8 / 4$ & $>128$ & 0.06 & CTX-M-14 \\
\hline 18 & $\mathrm{SCH}$ & ED & Blood & 2011-O4-O1 & $>128$ & $64 / 4$ & $>128$ & 0.06 & CTX-M-15 \\
\hline $19^{\mathrm{a}}$ & $\mathrm{SCH}$ & ED & Urine & 2011-04-07 & 64 & $8 / 4$ & $>128$ & $\leq 0.03$ & CTX-M-14 \\
\hline 20 & $\mathrm{SCH}$ & ED & Urine & $2011-04-20$ & $>128$ & $8 / 4$ & $>128$ & $\leq 0.03$ & CTX-M-15 \\
\hline 21 & $\mathrm{SCH}$ & ED & Ascites & $2011-04-30$ & 64 & $16 / 4$ & 128 & $\leq 0.03$ & CTX-M-15 \\
\hline 22 & $\mathrm{SCH}$ & ED & Pus & 2010-10-11 & $>128$ & $128 / 4$ & $>128$ & 0.5 & CTX-M-15 \\
\hline
\end{tabular}

MIC, minimum inhibitory concentration; ESBL, extended-spectrum $\beta$-lactamase; SMC, Samsung Medical Center; ED, Emergency Department; OPD, Out Patient Department; SCH, Samsung Changwon Hospital.

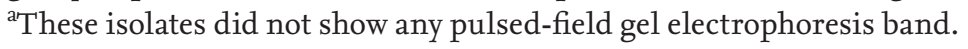

related. The remaining 10 isolates were classified into four closely related groups. Taking the PFGE results into consideration, it is reasonable to assume that the MDR-ESBL-EC isolates identified in this study belong to diverse clones.

Numerous studies have demonstrated that most ESBL-producing Enterobacteriaceae are resistant to multiple antibiotic classes. In addition to the agents included in our definition of MDR, we also noted a high prevalence of resistance to piperacillin/tazobactam and tobramycin among the MDR-ESBL-EC isolates $36.7 \%$ and $93.3 \%$, re- spectively).

The types of infection due to MDR-ESBL-EC were generally those expected for E. coli, with UTIs predominating, followed by intra-abdominal or pancreaticobiliary tract infections. FQs, TMP-SMX, and aminoglycosides are commonly used in community-onset infections, and if the infection is not severe, can be additional treatment options for UTIs.

We found a high prevalence of community-onset MDR-ESBL-EC isolates that also demonstrated resistance to additional antibiotics. Of great concern is the 
fact that one in four ESBL-EC isolates in this study was MDR. As the prevalence of MDR-ESBL-EC infections increases, inappropriate antibiotic treatment will also increase. A previous study demonstrated that increased mortality is not related to ESBL production but to the fact that the empirical therapies used were more frequently inappropriate among patients with ESBL-EC [5]. Reliance on carbapenems will continue to increase because other effective treatment options are increasingly limited. However, the increasing use of carbapenems has been paralleled by the rapid emergence of carbapenem resistance [11]. We suggest the empirical use of carbapenems only for patients with community-onset UTIs or intra-abdominal sepsis with any risk factor for MDR-ESBL-EC. However, other classes of antibiotics, especially amikacin and piperacillin/tazobactam, can be used for empirical therapy for patients with less severe infections caused by gram-negative bacilli, based on antimicrobial susceptibility results [34,35].

It has been shown previously that an ESBL-producing organism can be transferred to a hospital environment from the community [36]. Failure to consider the emergence of drug-resistant organisms in the community could undermine infection-control efforts in hospitals and render empirical antibiotic therapy inadequate. Interventions to limit the emergence of ESBL-producing isolates have traditionally focused on restricting certain antimicrobial agents, such as third-generation cephalosporins, associated with ESBL infections. Although such interventions are certainly an important component in efforts to control the emergence of ESBL-producing isolates, infection-control measures are also likely to be critical in interrupting the spread of such organisms.

There are several recognized limitations to our study. First, our study was observational, and thus, unknown risk factors might have been unequally distributed between the study groups. Harris et al. [37] suggested that using control patients infected with susceptible pathogens biases studies from the null hypothesis. Hence, we used a case-control-control study to assess the identifiable risk factors associated with community-onset MDR-ESBL-EC. Limitations related to the case-controlcontrol study design may also include the fact that the patients in CG II may not truly represent the population. We matched case and control patients on the basis of hospital and time period but intentionally did not match for variables such as age, sex, or severity of underlying conditions, which might themselves have been risk factors [38]. Second, not all ESBL-EC isolates were available for further molecular analysis. Therefore, the ESBL characterization results may not be representative of all ESBL-EC isolates. However, there was no systematic process affecting which isolates were not available and more than $91 \%$ of isolates were available for molecular analysis. Third, plasmid analysis was not done. Thus, the possibility of dissemination of MDR-ESBLEC isolates through the spread of such extrachromosomal genetic elements (even in the presence of unrelated PFGE patterns) cannot be excluded. Fourth, because previous exposure to FQs is likely to be associated with nosocomial acquisition, a well-established risk factor for resistance, our findings may have been affected by known or unknown confounders. Finally, our study was conducted in a large referral care medical center and a smaller community hospital. Thus, the results may not be generalizable to other types of institutions.

In conclusion, the emergence of MDR among community-onset ESBL-EC is particularly troublesome. We determined that $27.8 \%$ of ESBL-EC isolates, even in community-onset infections, were MDR and that the independent risk factors for infections caused by such isolates were prior exposure to $\mathrm{FQs}$, receipt of immunosuppressive therapy, and HCA infection. Clinicians should be aware of the occurrence of community-onset MDR-ESBL-EC among predisposed patients and select initial appropriate empirical antimicrobial therapy to reduce morbidity and mortality in such patients. Given that a limited number of new antimicrobial agents are being developed to treat MDR-ESBL-EC, rigorous infection control measures are advocated to prevent the spread of this microorganism. Also, an assessment of the effectiveness of strategies implemented to minimize the exposure to FQs in patients at risk for developing community-onset ESBL-EC infections would be of interest.

\section{KEY MESSAGE}

1. Among community-onset extended-spectrum $\beta$-lactamase-producing Escherichia coli (ESBL-EC) infections, one in four ESBL-EC isolates was multidrug resistance (MDR). 
2. The independent risk factors for communityonset MDR ESBL-EC infection were prior exposure to fluoroquinolones, receipt of immunosuppressive therapy, and healthcareassociated infection.

\section{Conflict of interest}

No potential conflict of interest relevant to this article was reported.

\section{Acknowledgments}

We thank Min Kyeong Cha and Jin Yang Baek for technical support. This study was supported by the Basic Research Program through the National Research Foundation of Korea (NRF) funded by the Ministry of Education, Science and Technology (No. 2010-0021572). Bacterial isolates were obtained from the Asian Bacterial Bank (ABB) of the Asia Pacific Foundation for Infectious Diseases (APFID).

\section{REFERENCES}

1. Jeong HS, Bae IK, Shin JH, et al. Prevalence of plasmidmediated quinolone resistance and its association with extended-spectrum beta-lactamase and AmpC betalactamase in Enterobacteriaceae. Korean J Lab Med 2011;31:257-264.

2. Paterson DL, Ko WC, Von Gottberg A, et al. International prospective study of Klebsiella pneumoniae bacteremia: implications of extended-spectrum beta-lactamase production in nosocomial Infections. Ann Intern Med 2004;140:26-32.

3. Pitout JD, Laupland KB. Extended-spectrum betalactamase-producing Enterobacteriaceae: an emerging public-health concern. Lancet Infect Dis 2008;8:159-166.

4. Paterson DL, Bonomo RA. Extended-spectrum beta-lactamases: a clinical update. Clin Microbiol Rev 2005;18:657686.

5. Rodriguez-Bano J, Picon E, Gijon P, et al. Community-onset bacteremia due to extended-spectrum beta-lactamaseproducing Escherichia coli: risk factors and prognosis. Clin Infect Dis 2010;50:40-48.

6. Gupta K, Hooton TM, Naber KG, et al. International clinical practice guidelines for the treatment of acute un- complicated cystitis and pyelonephritis in women: a 2010 update by the Infectious Diseases Society of America and the European Society for Microbiology and Infectious Diseases. Clin Infect Dis 2011;52:e103-e120.

7. Solomkin JS, Mazuski JE, Bradley JS, et al. Diagnosis and management of complicated intra-abdominal infection in adults and children: guidelines by the Surgical Infection Society and the Infectious Diseases Society of America. Clin Infect Dis 2010;50:133-164.

8. The Korean Society of Infectious Diseases Korean Society for Chemotherapy and The Korean Society of Clinical Microbiology. Clinical guideline for the diagnosis and treatment of gastrointestinal infections. Infect Chemother 2010;42:323-361.

9. Kang CI, Song JH, Chung DR, et al. Risk factors and treatment outcomes of community-onset bacteraemia caused by extended-spectrum beta-lactamase-producing Escherichia coli. Int J Antimicrob Agents 2010;36:284-287.

10. Hyle EP, Lipworth AD, Zaoutis TE, et al. Risk factors for increasing multidrug resistance among extendedspectrum beta-lactamase-producing Escherichia coli and Klebsiella species. Clin Infect Dis 2005;40:1317-1324.

11. Kritsotakis EI, Tsioutis C, Roumbelaki M, Christidou A, Gikas A. Antibiotic use and the risk of carbapenemresistant extended-spectrum-\{beta\}-lactamase-producing Klebsiella pneumoniae infection in hospitalized patients: results of a double case-control study. J Antimicrob Chemother 2011;66:1383-1391.

12. Park SY, Kang CI, Joo EJ, et al. Risk factors for multidrug resistance in nosocomial bacteremia caused by extended-spectrum beta-lactamase-producing Escherichia coli and Klebsiella pneumoniae. Microb Drug Resist 2012;18:518-524.

13. Serefhanoglu K, Turan H, Timurkaynak FE, Arslan H. Bloodstream infections caused by ESBL-producing E. coli and K. pneumoniae: risk factors for multidrug-resistance. Braz J Infect Dis 2009;13:403-407.

14. Rafailidis PI, Bliziotis IA, Falagas ME. Case-control studies reporting on risk factors for emergence of antimicrobial resistance: bias associated with the selection of the control group. Microb Drug Resist 2010;16:303-308.

15. Kang CI, Wi YM, Lee MY, et al. Epidemiology and risk factors of community onset infections caused by extended-spectrum beta-lactamase-producing Escherichia coli strains. J Clin Microbiol 2012;50:312-317.

16. Perl TM, Dvorak L, Hwang T, Wenzel RP. Long-term sur- 
vival and function after suspected gram-negative sepsis. JAMA 1995;274:338-345.

17. Friedman ND, Kaye KS, Stout JE, et al. Health care: associated bloodstream infections in adults: a reason to change the accepted definition of community-acquired infections. Ann Intern Med 2002;137:791-797.

18. Garner JS, Jarvis WR, Emori TG, Horan TC, Hughes JM. CDC definitions for nosocomial infections, 1988. Am J Infect Control 1988;16:128-140.

19. Bone RC, Balk RA, Cerra FB, et al. Definitions for sepsis and organ failure and guidelines for the use of innovative therapies in sepsis. The ACCP/SCCM Consensus Conference Committee. American College of Chest Physicians/ Society of Critical Care Medicine. Chest 1992;101:16441655.

20. Kim J, Lim YM, Jeong YS, Seol SY. Occurrence of CTXM-3, CTX-M-15, CTX-M-14, and CTX-M-9 extendedspectrum beta-lactamases in Enterobacteriaceae clinical isolates in Korea. Antimicrob Agents Chemother 2005;49:1572-1575.

21. Kim J, Lim YM, Rheem I, et al. CTX-M and SHV-12 betalactamases are the most common extended-spectrum enzymes in clinical isolates of Escherichia coli and Klebsiella pneumoniae collected from 3 university hospitals within Korea. FEMS Microbiol Lett 2005;245:93-98.

22. Chung DR, Lee HR, Lee SS, et al. Evidence for clonal dissemination of the serotype K1 Klebsiella pneumoniae strain causing invasive liver abscesses in Korea. J Clin Microbiol 2008;46:4061-4063.

23. Tenover FC, Arbeit RD, Goering RV, et al. Interpreting chromosomal DNA restriction patterns produced by pulsed-field gel electrophoresis: criteria for bacterial strain typing. J Clin Microbiol 1995;33:2233-2239.

24. Apisarnthanarak A, Kiratisin P, Saifon P, Kitphati R, Dejsirilert S, Mundy LM. Clinical and molecular epidemiology of community-onset, extended-spectrum beta-lactamase-producing Escherichia coli infections in Thailand: a case-case-control study. Am J Infect Control 2007;35:606-612.

25. Hsieh CJ, Shen YH, Hwang KP. Clinical implications, risk factors and mortality following community-onset bacteremia caused by extended-spectrum $\beta$-lactamase (ESBL) and non-ESBL producing Escherichia coli. J Microbiol Immunol Infect 2010;43:240-248.

26. Lopez-Dupla M, Martinez JA, Vidal F, et al. Previous ciprofloxacin exposure is associated with resistance to beta- lactam antibiotics in subsequent Pseudomonas aeruginosa bacteremic isolates. Am J Infect Control 2009;37:753758.

27. Villers D, Espaze E, Coste-Burel M, et al. Nosocomial Acinetobacter baumannii infections: microbiological and clinical epidemiology. Ann Intern Med 1998;129:182-189.

28. Giufre M, Graziani C, Accogli M, et al. Escherichia coli of human and avian origin: detection of clonal groups associated with fluoroquinolone and multidrug resistance in Italy. J Antimicrob Chemother 2012;67:860-867.

29. Kopterides P, Koletsi PK, Michalopoulos A, Falagas ME. Exposure to quinolones is associated with carbapenem resistance among colistin-susceptible Acinetobacter baumannii blood isolates. Int J Antimicrob Agents 2007;30:409-414.

30. Livermore DM. Of pseudomonas, porins, pumps and carbapenems. J Antimicrob Chemother 2001;47:247-250.

31. Pascual FE, Matthay MA, Bacchetti P, Wachter RM. Assessment of prognosis in patients with community-acquired pneumonia who require mechanical ventilation. Chest 2000;117:503-512.

32. Cho SY, Kang CI, Cha MK, et al. Clinical features and treatment outcomes of bloodstream infections caused by extended-spectrum $\beta$-lactamase-producing Escherichia coli sequence type 131. Microb Drug Resist 2015;21:463469.

33. Cha MK, Kang CI, Kim SH, et al. Comparison of the microbiological characteristics and virulence factors of ST131 and non-ST131 clones among extended-spectrum $\beta$-lactamase-producing Escherichia coli causing bacteremia. Diagn Microbiol Infect Dis 2016;84:102-104.

34. Park SH, Choi SM, Chang YK, et al. The efficacy of non-carbapenem antibiotics for the treatment of community-onset acute pyelonephritis due to extended-spectrum beta-lactamase-producing Escherichia coli. J Antimicrob Chemother 2014;69:2848-2856.

35. Rodriguez-Bano J, Navarro MD, Retamar P, Picon E, Pascual A; Extended-Spectrum Beta-Lactamases-Red Espanola de Investigacion en Patologia Infecciosa/Grupo de Estudio de Infeccion Hospitalaria Group. Beta-lactam/ beta-lactam inhibitor combinations for the treatment of bacteremia due to extended-spectrum beta-Lactamase-producing Escherichia coli: a post hoc analysis of prospective cohorts. Clin Infect Dis 2012;54:167-174.

36. Ben-Ami R, Schwaber MJ, Navon-Venezia S, et al. Influx of extended-spectrum beta-lactamase-producing enterobac- 
teriaceae into the hospital. Clin Infect Dis 2006;42:925934 .

37. Harris AD, Samore MH, Lipsitch M, Kaye KS, Perencevich E, Carmeli Y. Control-group selection importance in studies of antimicrobial resistance: examples applied to Pseudomonas aeruginosa, Enterococci, and Escherichia coli. Clin Infect Dis 2002;34:1558-1563.

38. Rodriguez-Bano J, Alcala JC, Cisneros JM, et al. Community infections caused by extended-spectrum beta-lactamase-producing Escherichia coli. Arch Intern Med 2008;168:1897-1902. 